\title{
BROAD LINE VARIATIONS IN NGC 5548
}

\author{
Edward I. Rosenblatt and Matthew A. Malkan \\ Department of Astronomy and Astrophysics \\ University of California, Los Angeles \\ Los Angeles, California 90024-1562
}

Since broad line variations can, in principle, constrain the structure and kinematics of the broad line region in active galaxies we have conducted a monitoring program of 20 Seyfert galaxies over a 5 year period in order to study broad line flux and profile changes. Included in our sample is the Seyfert 1.5 galaxy NGC 5548. Fifteen observations were taken from 1979 to 1984 mainly with the 60 " Palomar telescope and a SIT vidicon spectrograph. Measurements show (Fig. 1) that both the $\mathrm{H} \beta$ and $\mathrm{H} \gamma$ line flux varied by $200 \%$ and the continuum varied by $300 \%$. Furthermore, these changes were positively correlated as one would expect from photoionization by a central continuum source.

In contrast to ealier studies which made qualitative claims that the Balmer lines in NGC 5548 were "quite asymmetric" (Anderson 1971, also see Peterson 1987), we find $\mathrm{H} \beta$ to be usually only marginally blue asymmetric at the $10 \%$ level or less after correcting for $\mathrm{Fe}$ II and narrow $\mathrm{H} \beta$ contamination. We find, however, a rather pronounced $\mathrm{H} \beta$ profile variation in our data (Fig. 2). When $\mathrm{H} \beta$ and the continuum are strong the profile is more symmetric compared to epochs when the line is relatively weak which show an absence of blue line flux from wing to core. The red-half does not seem to vary significantly, if at all. Lastly, the difference spectrum between average high and low $\mathrm{H} \beta$ intensity states appears as one rather continuous profile without any naturally divisible components.

\section{References}

Anderson, K. S. 1971, Ap. J., 169:449

Peterson, B. M. 1987, Ap. J., 312:79 

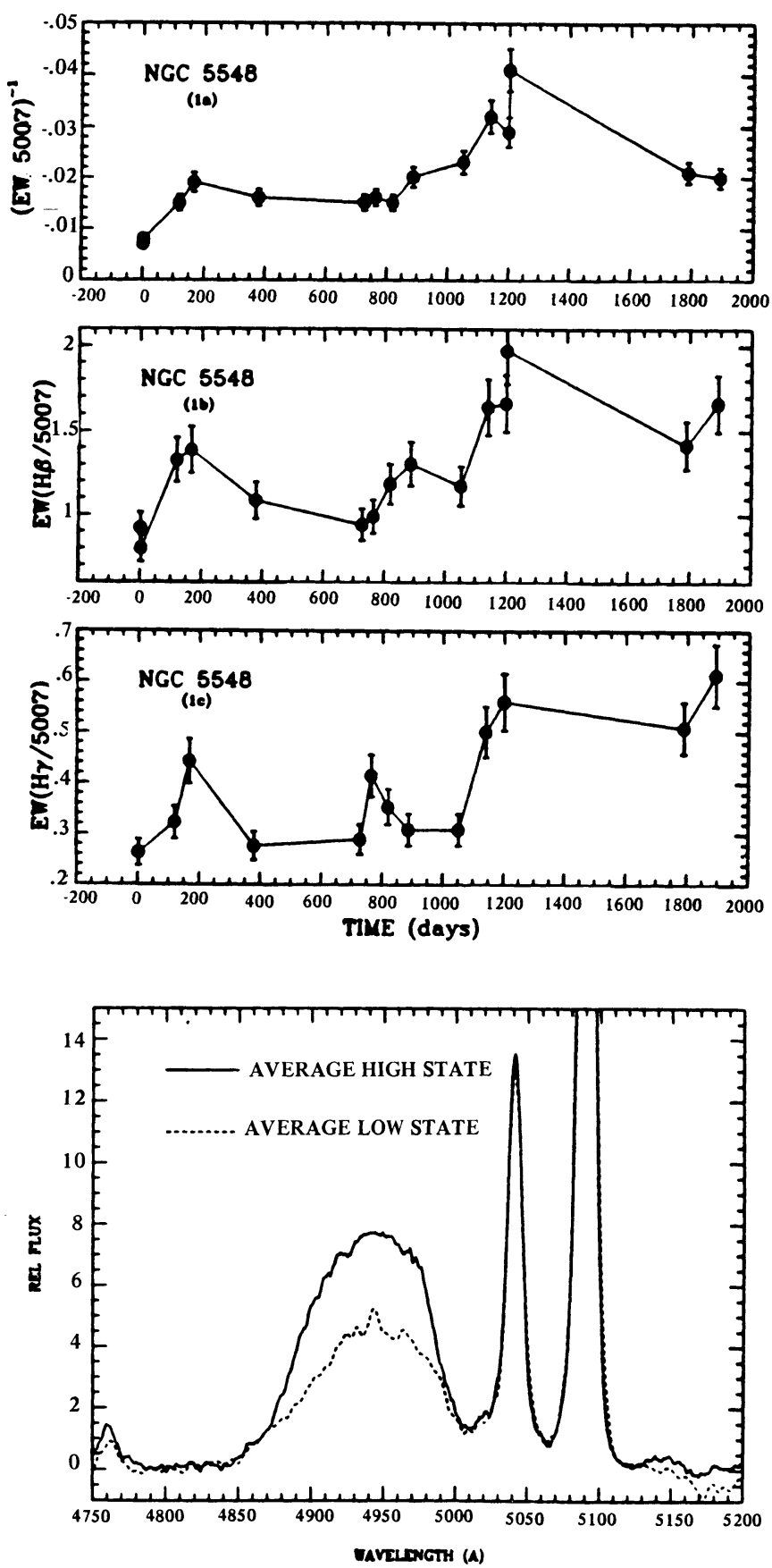

Figure 1. Light curves for NGC 5548 beginning $3 / 6 / 79$ and ending $6 / 6 / 84$. Assuming that $O[I I I] \lambda 5007$ is constant, equivalent width $(H \beta / 5007)$ and equivalent width $(H \gamma / 5007)$ represent the $H \beta$ and $H \gamma$ line intensities respectively, and the reciprocal of equivalent width $O[I I I] \lambda 5007$ represents the non-stellar continuum level. Note the temporal correlation between the Balmer line fluxs, which varied by a factor of two over this time period, and continuum flux which increased by a factor of three. $1 \sigma$ errors are $10 \%$.

Figure 2. Average spectra of all the high (solid line) and low (dashed line) relative strength $H \beta$ profiles for NGC 5548. Note the blue asymmetry found only in the low line flux $\boldsymbol{H} \beta$ profile. 